\title{
ON THE FOUNDATIONS OF CALCULUS OF VARIATIONS
}

\author{
BY \\ HERBERT BUSEMANN AND WALTHER MAYER
}

The subject of this paper will be variational problems $\int F(x, \dot{x}) d t=\min$ in parameter form with fixed endpoints. The existence of rectifiable minimizing arcs has been proved under exceedingly general conditions. However, as soon as one wants to establish differentiability properties of the solutions one uses the Euler equations and must therefore assume the existence of second partial derivatives of $F(x, \dot{x})$.

Hence it is not at all clear exactly which differentiability properties of the solutions are due to which properties of $F(x, \dot{x})$. The present paper tries to take a first step towards filling this gap. Simple examples show that without continuity or without the strict convexity of the indicatrix of $F(x, \dot{x})$ no general statements about the differentiability of the solution will be possible. Also, an example was given $\left.{ }^{1}\right)$ to show that even if these two conditions are satisfied, the minimizing curves are not necessarily of class $D^{\prime}$. In the example the indicatrix is an ellipse everywhere so that the variation of $F(x, \dot{x})$ for fixed $x$ is as smooth as possible. This and the example of the Minkowskian geometry (corresponding to an integrand not depending on the $x$ ) suggest investigation of the implications of the Lipschitz condition:

$$
|F(\bar{x}, \xi)-F(x, \xi)| \leqq C\left[\sum\left(\bar{x}_{i}-x_{i}\right)^{2}\right]^{1 / 2}\left[\sum\left(\xi^{i}\right)^{2}\right]^{1 / 2} .
$$

If the underlying space is two-dimensional we shall prove that already under this condition all minimizing arcs are continuously differentiable. Since we do not even require the existence of first partial derivatives of $F(x, \dot{x})$, our method has to be quite different from the usual ones. Our procedure was suggested by the treatment of geodesics on arbitrary convex surfaces in [4]. So far we have not succeeded in getting a similar result for more dimensional spaces. In the two-dimensional case three questions arise next-namely, whether under the same conditions the minimizing curves have second derivatives, whether every line element is contained in at least one minimizing arc, and whether a given line element occurs in at most one such arc. Simple examples will show that the answer to all three questions is negative.

We thought it useful for further investigations of this type to develop the general theory on a broader and more axiomatic base than the results mentioned would have required. Among the results which we get, the following may be of interest. If $F(x, \dot{x})>0$ for $\sum\left(\dot{x}_{i}\right)^{2}>0$ and continuous, then

Presented to the Society, September 12,1940; received by the editors May 22, 1940.

(1) See [3, pp. 281-285]. For the definition of classes $C^{\prime}$ and $D^{\prime}$, see $\$ 1$ below. 
$d(P, Q)=$ greatest lower bound of $\int_{D} F(x, \dot{x}) d t$, where $D$ varies over all oriented curves of class $D^{\prime}$ from $P$ to $Q$, defines a (not necessarily symmetric) metric space. The question arises how far $F(x, \dot{x})$ is determined by $d(P, Q)$. The answer is: $d(P, Q)$ does not change if, and only if, the convex closure of the indicatrix of the new function $F_{1}(x, \dot{x})$ at every point coincides with the convex closure of the indicatrix of $F(x, \dot{x})$. In particular, there is one and only one quasi-regular function $\bar{F}(x, \dot{x})$ to which $d(P, Q)$ belongs.

1. The space and its vector function. Our space $\mathfrak{M}$ will be an $n$-dimensional coordinate space of class 1 , i.e., it satisfies the following conditions:

(a) It is a Hausdorff space.

(b) Among the neighborhoods of a point $P$ there is at least one coordinate neighborhood (we shall abbreviate this as C.N.) $U(P)$, i.e., there exists a topological mapping $Q \rightarrow x=\left(x_{1}, \cdots, x_{n}\right)$ of $U(P)$ on to some sphere $\sum_{i=1}^{n} x_{i}^{2}<\rho$, $\rho>0$, of the $n$-dimensional Euclidean space. $U(P)$ together with this mapping forms the C.N. We call $x_{1}, \cdots, x_{n}$ the coordinates of $Q$. This requirement implies: When the intersection $U \cdot \bar{U}$ of two C.N. $U$ and $\bar{U}$ (of the same or different points) is not empty, then each point of $U \cdot \bar{U}$ has two sets of coordinates $x_{1}, \cdots, x_{n}$ and $\bar{x}_{1}, \cdots, \bar{x}_{n}$. In $U \cdot \bar{U}$ the $x_{i}$ are single valued and continuous functions of the $\bar{x}_{i}$ and conversely

$$
x_{i}=f_{i}(\bar{x}), \quad \bar{x}_{i}=\bar{f}_{i}(x) .
$$

(c) The functions $f_{i}(\bar{x})$ and $\bar{f}_{i}(\bar{x})$ have continuous first partial derivatives.

A parametrized curve $\mathfrak{S}$ in $\mathfrak{M}$ is defined in the following way: It is the continuous image of the interval $a \leqq t \leqq b$. It is oriented according to the orientation of $(a, b)$. If a certain subarc $\mathbb{S}^{\prime}: a^{\prime} \leqq t \leqq b^{\prime}$ of $\sqrt{ }$ is contained in a C.N. the arc $\left(\mathcal{S}^{\prime}\right.$ will have a representation $x_{i}=x_{i}(t), a^{\prime} \leqq t \leqq b^{\prime}$ where the functions $x_{i}(t)$ are continuous. If the functions $x_{i}(t)$ have continuous derivatives and if for no value $t_{0}$ all $\dot{x}_{i}\left(t_{0}\right)$ vanish, $\mathbb{S}^{\prime}$ will be called of class $C^{\prime}$, with respect to this C.N. If any subarc $\mathfrak{S}^{\prime \prime}$ of $\mathbb{S}^{\prime}$ is contained in a C.N. $U^{\prime \prime}$, the arc $\mathfrak{S}^{\prime \prime}$ will be of class $C^{\prime}$ with respect to $U^{\prime \prime}$. We can therefore say: We call $\mathfrak{E}$ of class $C^{\prime}$ if every subarc of $\mathbb{5}$ which is completely in one C.N. is of class $C^{\prime}$ with respect to this C.N.

Two parametrized curves $\sqrt{ }$ and $\mathfrak{S}^{\prime}$, defined for $a \leqq t \leqq b$ and $\alpha \leqq \tau \leqq \beta$ respectively, are said to belong to the same class if a topological mapping $t \rightarrow \tau(t)$ of $(a, b)$ onto $(\alpha, \beta)$ exists which preserves orientation and carries $\mathbb{E}$ into ${ }^{\prime}$. A class of parametrized curves is called a continuous curve. All curves of a class have the same initial and terminal points, which we therefore call the initial and terminal points of the continuous curve.

If $\sqrt{ } \mathfrak{F}$ is of class $C^{\prime}$ and if $\sqrt{ }$ can be transformed into $\mathfrak{S}^{\prime}$ by a mapping $t \rightarrow \tau(t)$ where $\tau$ is of class $C^{\prime}$ and $d \tau / d t>0$ for all $t$ in $(a, b)$, the curve $\mathcal{C}^{\prime}$ will be of class $C^{\prime}$. The parametrized curves $\widetilde{C}^{\prime}$ originating from $\subseteq$ in this way again form a class. We call this class a curve of class $C^{\prime}$ (or simply a $C^{\prime}$-curve). Statements involving the words "continuous curve" or " $C^{\prime}$-curve" will alwavs 
mean that they hold for all parametrized curves in the corresponding class.

A continuous curve will be called of class $D^{\prime}$ if it is the sum of a finite number of curves of class $C^{\prime}$.

Since $\mathfrak{M}$ is of class 1 , contravariant vectors are defined. A contravariant vector with origin $x$ and components $\lambda^{1}, \cdots, \lambda^{n}$ will be designated by $\left(x_{1}, \cdots, x_{n} ; \lambda^{1}, \cdots, \lambda^{n}\right)$ or in short by $(x, \lambda)$. If $\lambda^{j}=0$ for all $j$ we call the vector a null-vector. $\mathfrak{M}$ becomes a Finsler-manifold or Finsler-space as soon as a real function $F(x, \lambda)$ is defined for all contravariant vectors and has the following three properties:

(1a) $F(x, \lambda)$ is continuous as a function of the $2 n$ variables $x_{i}, \lambda^{j}$ when $x_{j}$ varies in a C.N.

(1b) $F(x, \lambda)$ is positive definite, i.e., positive if $(x, \lambda)$ is no null-vector.

(1c) $F(x, \lambda)$ is positively homogeneous of order 1 in $\lambda$, i.e., $F(x, c \lambda)$ $=c F(x, \lambda)$ for $c>0$.

It follows from (a) and (c) that $F$ vanishes for every null-vector. We do not require that $F(x, \lambda)=F(x,-\lambda)$. A neighborhood of $P$ which together with its closure is contained in a C.N. will be called a $k$-neighborhood (K.N.) of $P$. From $(1 \mathrm{a}, \mathrm{b}, \mathrm{c})$ we get the following useful

Lemma 1. For every $K . N . U(P)$ of $P$ two positive numbers $A$ and $B$, depending on the coordinate system, can be formed such that

$$
\begin{aligned}
A|\lambda| & \geqq F(x, \lambda) \geqq B|\lambda|, \\
A \max _{i}\left|\lambda^{i}\right| & \geqq F(x, \lambda) \geqq B \max _{i}\left|\lambda^{i}\right| .
\end{aligned}
$$

$\left|\lambda^{i}\right|$ designates, of course, the absolute value of $\lambda^{i}$; but $|\lambda|=\left(\sum\left(\lambda^{i}\right)^{2}\right)^{1 / 2}$ and more generally $|a|$ designates $\left(\sum a_{i}^{2}\right)^{1 / 2}$ when $a_{1}, \cdots, a_{m}$ is a set of numbers with known subscripts.

Let $\bar{C}_{P Q}$ be an oriented parametrized curve of class $D^{\prime}$ from $P$ to $Q$, $a \leqq t \leqq b$. Then in each of its points with a finite number of exceptions the contravariant tangential vector $\dot{x}_{i}(t)$ is defined and $F(x(t), \dot{x}(t))$ will be a piecewise continuous function. Hence

$$
I\left(\bar{C}_{P Q}\right)=\int_{a}^{b} F(x, \dot{x}) d t
$$

is positive and finite and will have the same value for all parametrized curves which belong to the same class $C_{P Q}$ as $\bar{C}_{P Q}$. We may therefore write $I\left(C_{P Q}\right)$ instead of $I\left(\bar{C}_{P Q}\right)$. As $F$-distance $d(P, Q)$ we define the greatest lower bound of $I\left(C_{P Q}\right)$ for all oriented curves of class $D^{\prime}$ from $P$ to $Q$. We have

(3a) $d(P, Q)>0$ for $P \neq Q, d(P, P)=0$.

(3b) $d(P, Q)+d(Q, R) \geqq d(P, R)$.

(3c) $d(P, Q)$ is continuous in the product space $\mathfrak{M} \times \mathfrak{M}$.

Finally we require the finite $F$-compactness of $\mathfrak{M}$ : 
(1d) If the distances $d\left(X_{\nu}, P\right)$ or $d\left(P, X_{\nu}\right)$ are bounded, then the sequence $X_{\nu}, \nu=1,2, \cdots$, has an accumulation point.

By $\mathfrak{U}_{\rho}(P)$ and $\mathfrak{W}_{\rho}(P)$ we designate the sets of points $X$ with

$$
d(P, X)<\rho, \quad d(X, P)<\rho,
$$

respectively. If $P$ and $\rho$ vary, the $\mathfrak{u}_{\rho}(P)$ will traverse a system of neighborhoods equivalent to the system of all neighborhoods, and so will the $\mathfrak{B}_{\rho}(P)$.

A curve $C_{P Q}$ from $P$ to $Q$ of class $D^{\prime}$ will be called minimizing if $I\left(C_{P Q}\right)$ $=d(P, Q)$. If $R, S, T$ are any three points which lie in this order on $C_{P Q}$ we have

$$
d(R, S)+d(S, T)=d(R, T) .
$$

An oriented continuous curve from $P$ to $Q$ on which (4) holds for any three points which occur in this order on $C_{P Q}$ will be called a Hilbert curve $\left.{ }^{2}\right)$. A Hilbert curve is necessarily simple (a Jordan arc); therefore we also call it a Hilbert arc. By the well known procedure of successive construction of mid-points one shows that for any pair of points $P, Q$ there exists a Hilbert arc from $P$ to $Q$.

Let $\bar{C}_{P Q}=P(t), a \leqq t \leqq b$, be any parametrized curve from $P$ to $Q$, $a=t_{0}<t_{1}<\cdots<t_{n}=b$ a subdivision of $(a, b)$. We form

$$
S_{m}=\sum_{i=0}^{m-1} d\left(P_{i}, P_{i+1}\right)
$$

and call $L\left(\bar{C}_{P Q}\right)$ the least upper bound of $S_{m}$ as $\left(t_{0}, \cdots, t_{m}\right)$ varies over all subdivisions of $(a, b)$. It follows in the usual way from $(3 \mathrm{a}, \mathrm{b}, \mathrm{c})$ that $S_{m}$ will tend to $L\left(\bar{C}_{P Q}\right)$ whenever $\left(t_{0}, \cdots, t_{m}\right)$ traverses a sequence of subdivisions for which $\max _{i}\left(t_{i+1}-t_{i}\right)$ tends to zero. $L\left(\bar{C}_{P Q}\right)$ will have the same value for all parametrized curves which are in the same class $C_{P Q}$ as $\bar{C}_{P Q}$. We may therefore write $L\left(C_{P Q}\right)$ instead of $L\left(\bar{C}_{P Q}\right)$ which we call the generalized length of $C_{P Q}$. It follows from (4) that for Hilbert $\operatorname{arcs} H_{P Q}$

$$
L\left(H_{P Q}\right)=d(P, Q) .
$$

Conversely, if (5) is satisfied, $H_{P Q}$ will be a Hilbert curve.

We conclude this section with a remark which will be useful later. Let the conditions of Lemma 1 be satisfied and let $C_{P Q}$ be a curve of class $D^{\prime}$ in the K.N. $U(P)$. It follows from (2) that

$$
A E\left(C_{P Q}\right) \geqq I\left(C_{P Q}\right) \geqq B E\left(C_{P Q}\right),
$$

where $E(C)$ designates generally the Euclidean length of the curve $C$, which is defined since we move in a definite C.N.

(2) The words "Hilbert curve," as used here, correspond to the words "minimizing arc" as used in the introduction. 
Let now $U^{\prime}(P) \subset U(P)$ be chosen in such a way that both the Euclidean and the $F$-distance of any point $Q$ of $U^{\prime}(P)$ from the boundary of $U(P)$ are greater than the respective distance of $Q$ from any other point in $U^{\prime}(P)$. Then a Hilbert arc and the Euclidean segment $e_{R S}$ connecting two points $R, S$ of $U^{\prime}(P)$ will be contained in $U(P)$, hence we get from (6)

$$
A E\left(e_{R S}\right) \geqq d(R, S) \geqq B E\left(e_{R S}\right) ;
$$

and whenever we have two sequences of curves $C_{R S}^{m}$ and $K_{R S}^{m}$ with $E\left(C_{R S}^{m}\right)$ $\rightarrow E\left(e_{R S}\right)$ and $I\left(K_{R S}^{m}\right) \rightarrow d(R, S)$ the curves $C_{R S}^{m}$ and $K_{R S}^{m}$ will be contained in $U(P)$ from a certain subscript $m$ on. It also follows from (7) that curves with finite generalized length are rectifiable and conversely.

2. The indicatrix. We shall now study the function $F(x, \xi)=f(\xi)$ at a fixed point $P$ with coordinates $x$ in a fixed K.N. $P$ will then be the origin of the space of the contravariant vectors $\xi$. The hypersurface $f(\xi)=1$, the so-called indicatrix $\gamma(x)$ of $F(x, \xi)$ at $x$, determines $f(\xi)$ on account of its homogeneity. By

$$
\frac{\xi^{i}}{|\xi|} \rightarrow \frac{\xi^{i}}{f(\xi)}, \quad i=1, \cdots, n,
$$

we map the indicatrix topologically onto the unit sphere $|\xi|=1$. The inequality (1.2) gives us

$$
A|\xi| \geqq f(\xi) \geqq B|\xi|,
$$

and for the points $\xi$ on the indicatrix $\gamma(x)$

$$
1 / B \geqq|\xi| \geqq 1 / A,
$$

so that $\gamma(x)$ is between the spheres of radii $1 / A$ and $1 / B$ around the origin $x$.

Let $a_{i}$ be a covariant vector with origin $P=x$. Then $a_{i} \xi^{i}=c$ represents a hyperplane in $\xi$-space. For fixed $a_{i}$ the function $\phi(\xi)=a_{i} \xi^{i}$ is continuous on $\gamma(x)$ and has a positive maximum. For choosing $\xi^{i}=\rho a_{i}$ with a suitable $\rho>0$ we get from $\left(2^{\prime}\right)$

$$
\max \phi(\xi) \geqq|a| / A .
$$

If $* \xi$ and ${ }^{* *} \xi$ are two points at which this maximum is reached, the equations

$$
a_{i}\left(\xi^{i}-*^{*} \xi^{i}\right)=0, \quad a_{i}\left(\xi^{i}-* * \xi^{i}\right)=0
$$

represent the same plane $\pi$, which is called a supporting plane of $\gamma(x)$ (at each of the points of $\gamma \cdot \pi$ ). For each $* \xi$ in $\gamma \pi$ we have, on account of (3),

$$
a_{i}^{*} \xi^{i} \geqq|a| / A \text {. }
$$

For an arbitrary point $\xi$ of $\gamma(x)$ we have

$$
a_{i} \xi^{i} \leqq a_{i}^{*} \xi^{i}
$$


and therefore $a_{i}\left(\xi^{i}-{ }^{*} \xi^{i}\right) \leqq 0$, where the equality only holds for $\xi \subset \gamma \cdot \pi$. If $\pi$ is any plane not containing the origin, we call $\pi^{\prime}$ the closed half-space bounded by $\pi$ which contains it. Then $\gamma(x)$ and also the domain $\Gamma: f(\xi) \leqq 1$ bounded by $\gamma$ are completely contained in the half-space $\pi^{\prime}$. This shows that our definition of a supporting plane coincides with the usual one. We have

If $\pi: a_{i}\left(\xi^{i}-{ }^{*} \xi^{i}\right)=0,{ }^{*} \xi \subset \gamma \cdot \pi$, is a supporting plane of $\gamma$, then

$$
f(\eta)-f(* \xi) \geqq 0 \quad \text { if } a_{i}\left(\eta^{i}-{ }^{*} \xi^{i}\right) \geqq 0 ;
$$

and conversely, if $f\left({ }^{*} \xi\right)=1$ and if $(4)$ holds, $a_{i}\left(\xi^{i}-{ }^{*} \xi^{i}\right)=0$ will be a supporting plane of $\gamma$ (or $\Gamma$ ) at $* \xi$.

If we replace $a_{i}$ by $c a_{i}, c>0$, the supporting plane $\pi$ will not change. $\left(3^{\prime}\right)$ shows that we can find a $\kappa>0$ such that with $A_{i}=\kappa a_{i}$

$$
A_{i}^{*} \xi^{i}=f\left({ }^{*} \xi\right)
$$

for every vector $* \xi$ in $\gamma \pi$. (5) will hold for any vector $c \xi^{i}, c>0$, if it holds for $\xi$.

We call $A_{i}$ the normalized normal of $\pi$ and every vector $\xi \neq 0$ which satisfies (5) a supporting vector belonging to $A_{i}$. Among these supporting vectors those with $f(* \xi)=1$ are called normalized.

We can now say

Theorem 1. If $A_{i}$ is a normalized normal of $\pi, \xi^{i}$ a fixed supporting vector belonging to $A_{i}$, then

$$
f(\eta)-f(\xi)-A_{i}\left(\eta^{i}-\xi^{i}\right) \geqq 0,
$$

and the equality sign holds if, and only if, $A_{i}\left(\eta^{i}-\xi^{i}\right)=0$.

Remark. Replacing $f(\eta)$ and $f(\xi)$ by $F(x, \eta)$ and $F(x, \xi)$ one recognizes in the left side of (6) a generalization of the Weierstrass $\varepsilon$-function.

Proof. With the help of (5) we reduce (6) to

$$
f(\eta)-A_{i} \eta^{i} \geqq 0 \text {. }
$$

The left side being homogeneous in the $\eta^{i},\left(6^{\prime}\right)$ will be proved if it is true for all $\eta^{i}$ for which $f(\eta)=f(\xi)$; hence it is sufficient to prove $(6)$ for $f(\eta)=f(\xi)$. But then (6) reduces to $-A_{i}\left(\eta^{i}-\xi^{i}\right) \geqq 0$ or

$$
A_{i}\left(\frac{\eta^{i}}{f(\eta)}-\frac{\xi^{i}}{f(\xi)}\right) \leqq 0
$$

and now $\eta^{i} / f(\eta)$ is an arbitrary vector on $\gamma, \pi$ a supporting plane at $\xi^{i} / f(\xi)$, and the theorem follows from our previous results.

The surface $f(\xi)=1$ will be (strictly) convex, if the set $f(\xi) \leqq 1$ is (strictly) convex, and conversely. We quote the following known theorem (compare $[2$, pp. 21, 22]).

The indicatrix $f(\xi)=1$ is convex if, and only if, 


$$
f(\xi+\eta) \leqq f(\xi)+f(\eta)
$$

for arbitrary contravariant vectors $\xi^{i}$ and $\eta^{i}$. The convexity will be strict if, and only if, in (7) the equality sign holds only for

$$
\lambda \xi^{i}=\mu \eta^{i}, \quad \lambda \geqq 0, \quad \mu \geqq 0, \quad \lambda+\mu>0 .
$$

If the indicatrix of $F$ at $x$ is (strictly) convex in one coordinate system, it will be (strictly) convex in every coordinate system.

If $\gamma$ is not convex we consider its convex closure $\bar{\Gamma}$ which is the same as the convex closure of $\Gamma$. By $\bar{\gamma}(x)$ we designate the boundary of $\bar{\Gamma}$. We shall make use of the following fact (cf. [2, p. 6]).

Every supporting plane of $\gamma(x)$ is a supporting plane of $\bar{\gamma}(x)$ and (which is the less trivial statement) every supporting plane of $\bar{\gamma}(x)$ is a supporting plane of $\gamma(x)$.

Let now $\eta^{i} \neq 0$ be any vector with $f(\eta)=1$ and denote by $r(\eta)$ the ray carrying $\eta$. Consider the intersections $\beta \eta^{i}, \beta>0$, of the different supporting planes of $\gamma$ with $r(\eta)$. We have $\beta \geqq 1$. Let $\alpha(\eta) \eta^{i}$ be the point where $\bar{\gamma}(x)$ hits $r(\eta)$ and define

$$
\alpha(x, c \eta)=\alpha(\eta) \quad \text { for } c>0,
$$

then

$$
\alpha(x, \xi) \geqq 1 \quad \text { for all } x \text { and } \xi
$$

and we have

$$
\alpha(\eta)=\min \beta .
$$

For since a supporting plane of $\bar{\gamma}$ at $\alpha(\eta) \eta^{i}$ is a supporting plane of $\gamma, \alpha(\eta)$ belongs to the numbers $\beta$. There can be no $\beta<\alpha(\eta)$ since the corresponding supporting plane of $\gamma(x)$ would separate $\alpha(\eta) \eta^{i}$ from the origin $x$ (that is $\xi=0$ ), and could therefore be no supporting plane of $\bar{\gamma}(x)$.

Since $\gamma$ is bounded and closed, and $\alpha(\eta) \eta^{i}$ belongs to the convex closure of $\gamma$, we can find points $\xi_{1}^{i}, \cdots, \xi_{n}^{i}$ on $\gamma$ such that $\alpha(\eta) \eta^{i}$ belongs to the simplex with vertices $\xi_{1}^{i}, \cdots, \xi_{n}^{i}$ (cf. [2, p. 9]). For later use we formulate this statement as follows:

TheOREM 2. Given a contravariant vector $(x, \eta) \neq(x, 0)$ one can find contravariant vectors $\left(x, \xi_{1}\right), \cdots,\left(x, \xi_{n}\right)$ with

$$
F(x, \eta)=F\left(x, \xi_{1}\right)=\cdots=F\left(x, \xi_{n}\right)
$$

such that

$$
\alpha(x, \eta) \cdot(x, \eta)=\sum_{\alpha=1}^{n} p_{\alpha} \cdot\left(x, \xi_{\alpha}\right), \quad p_{\alpha} \geqq 0, \quad \sum_{\alpha=1}^{n} f_{\alpha}=1
$$

We now define a new function $\bar{F}(x, \xi)$ by 


$$
\bar{F}(x, \xi)=\bar{f}(\xi)=\frac{1}{\alpha(x, \xi)} F(x, \xi)=\frac{1}{\alpha(\xi)} f(\xi) .
$$

$\bar{f}(\xi)$ like $f(\xi)$ is positive definite and positive homogeneous of order 1 in $\xi$. We see from $(8 b)$ that

$$
\bar{F}(x, \xi) \leqq F(x, \xi) .
$$

$\bar{F}(\alpha, \xi)=1$ is the equation of $\bar{\gamma}=\bar{\gamma}(x)$, since $\alpha(\xi) \xi^{i}$ is on $\bar{\gamma}$ with $\xi^{i}$ on $\gamma$. With $\gamma$ the surface $\bar{\gamma}$ is between the spheres of radii $1 / A$ and $1 / B$, i.e., we still have

$$
A|\xi| \geqq \bar{f}(\xi) \geqq B|\xi|
$$

and for $\xi \subset \bar{\gamma}$

$$
1 / B \geqq|\xi| \geqq 1 / A \text {. }
$$

The indicatrix $\gamma$ will be convex if, and only if, $\bar{f}(\eta) \equiv f(\eta)$ or $\alpha(\eta) \equiv 1$. We prove

THEOREM 3. $\bar{F}(x, \eta)$ is a continuous function of the $2 n$ variables $x_{1}, \cdots, x_{n}$, $\eta^{\prime}, \cdots, \eta^{n}$. Therefore $\alpha(x, \eta)$ is also continuous.

Proof. Let $\eta_{m} \rightarrow \eta_{0} \neq 0, x_{m} \rightarrow x_{0}$. Let $P_{m}$ be the point at which the ray carrying $\eta_{m}$ intersects $\bar{\gamma}\left(x_{m}\right)\left(P_{m}\right.$ is the point $\alpha\left(x_{m}, \eta_{m}\right) \cdot\left(\eta_{m}^{i} / F\left(x_{m}, \eta_{m}\right)\right)$ in the vector space with origin $\left.x_{m}\right)$. Let $\pi_{m}$ be a supporting plane of $\bar{\gamma}\left(x_{m}\right)$ at $P_{m} . \pi_{m}$ is also a supporting plane of $\gamma\left(x_{m}\right)$ at some point $Q_{m}$. We may assume that $Q_{m}$ converges toward a point $Q$ and $\pi_{m}$ toward a plane $\pi$ at $x_{0}$. (Hereby we make use of the fact that a sequence will converge toward $P$, if every subsequence contains a subsequence which converges to $P$.) Then $P_{m}$ will converge to the intersection $P$ of $\pi$ with the ray $r\left(\eta_{0}\right)$ issuing from $x_{0}$. We have to prove that $P$ is the point where $r\left(\eta_{0}\right)$ intersects $\bar{\gamma}\left(x_{0}\right)$, i.e., the point $\alpha\left(x_{0}, \eta_{0}\right)\left(\eta_{i} / F\left(x_{0}, \eta_{0}\right)\right)$. Since $F(x, \eta)$ is continuous $Q$ will be on $\gamma\left(x_{0}\right)$. Since $\pi$ passes through $Q$ it is a supporting plane of $\gamma\left(x_{0}\right)$ at $Q$. Otherwise $\pi$ would separate $x_{0}$ from a point $\bar{\eta}^{i}$ of $\gamma\left(x_{0}\right)$ and also from all points of a certain closed sphere $\sigma: \sum\left(\xi^{i}-\bar{\eta}^{i}\right)^{2} \leqq \rho$. For large $m$ the plane $\pi_{m}$ would also separate the sphere from the origin $x_{m}$, but $\sigma$ would contain points of $\gamma\left(x_{m}\right)$.

Since $\pi$ is a supporting plane of $\gamma\left(x_{0}\right)$ and passes through $P$ we see from $\left(8^{\prime}\right)$ that $P$ is either outside or on $\bar{\gamma}\left(x_{0}\right)$. If $P$ was outside of $\bar{\gamma}\left(x_{0}\right)$ we could find a plane $\pi^{*}$ separating a certain closed sphere $\sigma^{*}$ around $P$ from $\bar{\gamma}\left(x_{0}\right)$ (see [2, p. 4]). For large $m$, the plane $\pi^{*}$ would also separate $\sigma^{*}$ from $\gamma\left(x_{m}\right)$ and therefore $P_{m}$ from $\gamma\left(x_{m}\right)$, hence $P_{m}$ could not belong to the convex closure of $\gamma\left(x_{m}\right)$, but it lies on $\bar{\gamma}\left(x_{m}\right)$.

Remark on Theorem 3. The proof contains the well known fact:

If $\gamma\left(x_{m}\right)$ is convex and $\gamma\left(x_{m}\right) \rightarrow \gamma\left(x_{0}\right)$ and if $\pi_{m}$ is a supporting plane of $\gamma\left(x_{m}\right)$, then each accumulation plane of the $\pi_{m}$ is a supporting plane of $\gamma\left(x_{0}\right)$.

If the indicatrix $\gamma(x)$ is strictly convex, a supporting plane $\sum a_{i} \eta^{i}=c>0$ 
can have only one common point with $\gamma(x)$. Hence, given a covariant vector $a_{i} \neq 0$ there is only one normalized supporting vector $\xi^{i}$ belonging to $\alpha_{i}$; $\xi^{i}$ is a single-valued function of $a_{i}$ with

$$
\xi^{i}\left(\lambda a_{i}\right)=\xi^{i}(a)
$$

for $\lambda>0$.

If, conversely, $\xi^{i}$ is a point on $\gamma(x)$, a supporting plane $\sum a_{i} \eta^{i}=c>0$ through $\xi$ exists; hence $\xi$ belongs to $a$, but $a$ will in general not be uniquely determined.

We call $F(x, \xi)$ quasi-regular at $x$ in the direction $\xi$, or $\xi$ a quasi-regular direction of $F$ at $x$ if $\alpha(x, \xi)=1$. If $\xi$ is quasi-regular, supporting planes of $\gamma(x)$ through $\xi$ will exist. If each such supporting plane meets $\gamma$ only at $\xi$, the direction $\xi$ is called regular. If all directions of $F(x, \xi)$ at $x$ are quasi-regular (regular), $F(x, \xi)$ is called quasi-regular (regular) at $x$. We have that $F(x, \xi)$ is (regular) quasi-regular at $x$ if, and only if, the indicatrix $F(x, \xi)=1$ is (strictly) convex.

We shall later make essential use of the

TheOREM 4. If $F(x, \xi)$ is regular at all points of a K.N., the functions

$$
\xi^{i}=\xi^{i}(x, a)
$$

are defined and single-valued for $q_{i} \neq 0$ and continuous in the $2 n$ variables $x_{1}, \cdots, x_{n}, a_{1}, \cdots, a_{n}$.

If $F$ is regular, it follows from the last remark that $F(x, \xi)=1$ is strictly convex, and therefore $\xi^{i}(x, e)$ is defined and single-valued for $q_{i} \neq 0$. Now let $x_{m} \rightarrow x_{0}$ and $a_{m} \rightarrow a_{0}$. There is exactly one supporting plane $\pi_{m}$ of $F\left(x_{m}, \eta\right)=1$ of the form $\sum a_{m, i} \eta^{i}=c_{m}>0$. This plane has only the point $\xi_{m}=\xi\left(x_{m}, a_{m}\right)$ in common with $\gamma\left(x_{m}\right)$. Each accumulation plane $\pi$ of the $\pi_{m}$ is a supporting plane of $F\left(x_{0}, \eta\right)=1$ according to the Remark on Theorem 3. Now the normals $a_{m}$ of $\pi_{m}$ converge to $a_{0}$; therefore each such $\pi$ has the form $\sum a_{0, i} \eta^{i}=c_{0}>0$. But there is only one supporting plane $\pi_{0}$ of $\gamma\left(x_{0}\right)$ of this form; hence the $\pi_{m}$ converge to $\pi_{0}$. The accumulation points of the $\xi_{m}$ must be in $\pi_{0} \cdot \gamma\left(x_{0}\right)$, but since $\gamma\left(x_{0}\right)$ is strictly convex $\pi_{0} \cdot \gamma\left(x_{0}\right)$ consists of exactly one point $\xi_{0}$; therefore, $\xi_{m} \rightarrow \xi_{0}=\xi\left(x_{0}, a_{0}\right)$, which completes the proof.

3. The relation between the $F$-metric and the $\bar{F}$-metric. The functions $F(x, \xi)$ and $\bar{F}(x, \xi)$ are both continuous as functions of the $2 n$ variables in a certain C.N. $U$ and for all $\xi$. If $\kappa$ is a compact subset of $U$ and $\sigma$ the unit sphere $|\xi|=1$, the product $\kappa \times \sigma$ will be compact; hence $F$ and $\bar{F}$ will be uniformly continuous on $\kappa \times \sigma$. We shall apply this fact in the following form.

Given an $\epsilon>0$ we can find a constant $\delta>0$ such that for $x, \bar{x} \subset \kappa ; \xi,{ }^{*} \xi \subset \sigma$; and $|x-\bar{x}|<\delta,|\xi-* \xi|<\delta$ the inequalities

$$
|F(\bar{x}, * \xi)-F(x, \xi)|<\epsilon, \quad|\bar{F}(\bar{x}, * \xi)-\bar{F}(x, \xi)|<\epsilon
$$

hold 
If $C_{P Q}$ is a curve of class $D^{\prime}$ from $P$ to $Q, x(t), a \leqq t \leqq b$, a representation of it, we put

$$
\bar{I}\left(C_{P Q}\right)=\int_{a}^{b} \bar{F}(x, \dot{x}) d t
$$

and call it the $\bar{F}$-length of $C_{P Q}$. Since $\bar{F} \leqq F$ we have

$$
\bar{I}\left(C_{P Q}\right) \leqq I\left(C_{P Q}\right) .
$$

Calling $h(P, Q)$ the greatest lower bound of $\bar{I}\left(C_{P Q}\right)$ for all curves of class $D^{\prime}$ leading from $P$ to $Q$ we have

$$
h(P, Q) \leqq d(P, Q) .
$$

The main purpose of this section is to prove that the equality holds. It will be sufficient to see that for a given $\epsilon^{*}>0$ and a given curve $C_{P Q}$ of class $D^{\prime}$ a $C_{P Q}^{*}$ of the same class exists with

$$
\left|\bar{I}\left(C_{P Q}\right)-I\left(C_{P Q}^{*}\right)\right|<\epsilon^{*} .
$$

$C_{P Q}$ can be decomposed into a finite number of arcs each of which is covered by one C.N.; we may therefore restrict ourselves to proving (4) for each such arc.

Now let $P(t), a \leqq t \leqq b$, be a parametrized representation of $C_{P Q}$ and $\pi_{m}=\left(a=t_{0}<t_{1}<\cdots<t_{m}=b\right)$ a subdivision of $(a, b)$ with $x_{i}$ the coordinates of $P_{i}=P\left(t_{i}\right)$. We form

$$
P_{m}=\sum_{k=0}^{m-1} F\left(x_{k}, \Delta x_{k}\right), \quad \quad \bar{P}_{m}=\sum_{k=0}^{m-1} \bar{F}\left(x_{k}, \Delta x_{k}\right)
$$

and

$$
P_{m}^{\prime}=\sum_{k=0}^{m-1} \int_{e P_{k} P_{k+1}} F(x, \dot{x}) d t, \quad \bar{P}_{m}^{\prime}=\sum_{k=1}^{m-1} \int_{e P_{k} P_{k+1}} \bar{F}(x, \dot{x}) d t
$$

where $\Delta x_{k}=x\left(t_{k+1}\right)-x\left(t_{k}\right)$ and $e_{R S}$ designates the oriented Euclidean segment from $R$ to $S$. It is known (see [1, pp. 286-289]) that for a given $\epsilon_{1}>0$ a $\delta_{1}>0$ can be found such that if $\max _{k}\left(t_{k+1}-t_{k}\right)<\delta_{1}$ one has

$$
\begin{array}{ll}
\left|I\left(C_{P Q}\right)-P_{m}\right|<\epsilon_{1}, & \left|I\left(C_{P Q}\right)-P_{m}^{\prime}\right|<\epsilon_{1}, \\
\left|\bar{I}\left(C_{P Q}\right)-\bar{P}_{m}\right|<\epsilon_{1}, & \left|\bar{I}\left(C_{P Q}\right)-\bar{P}_{m}\right|<\epsilon_{1},
\end{array}
$$

and therefore also

$$
\left|P_{m}-P_{m}^{\prime}\right|<2 \epsilon_{1}, \quad\left|\bar{P}_{m}-\bar{P}_{m}^{\prime}\right|<2 \epsilon_{1} .
$$

For $\Delta x_{k} \neq 0$ we put 


$$
\eta^{i}=\frac{\Delta x_{k, i}}{\alpha\left(x_{k}, \Delta x_{k}\right)}=\frac{\Delta x_{k, i}}{\alpha\left(x_{k}, \eta\right)}
$$

According to Theorem 2.2 we can find vectors $\xi_{1}, \cdots, \xi_{n}$ with

$$
F\left(x_{k}, \eta\right)=F\left(x_{k}, \xi_{1}\right)=\cdots=F\left(x_{k}, \xi_{n}\right)
$$

such that

$$
\alpha\left(x_{k}, \eta\right) \eta^{i}=\sum_{\alpha=1}^{n} p_{\alpha} \xi_{\alpha}^{i} \text { with } p_{\alpha} \geqq 0 \text { and } \sum p_{\alpha}=1
$$

We then have (see 2.10)

$$
\bar{F}\left(x_{k}, \Delta x_{k}\right)=\bar{F}\left(x_{k}, \alpha\left(x_{k}, \eta\right) \eta\right)=F\left(x_{k}, \eta\right)
$$

and

$$
\sum_{\alpha=1}^{n} F\left(x_{k}, p_{\alpha} \xi_{\alpha}^{i}\right)=F\left(x_{k}, \eta\right)
$$

Hence

$$
\bar{F}\left(x_{k}, \Delta x_{k}\right)=\sum_{\alpha=1}^{n} F\left(x_{k}, p_{\alpha} \xi_{\alpha}\right)
$$

where $\Delta x_{k}=\sum_{\alpha=1}^{n} p_{\alpha} \xi_{\alpha}$.

$\sum p_{\alpha} \xi_{\alpha}$ may be looked at as a polygon with oriented sides $\xi_{\alpha}$. Since $x_{k}$ is fixed in (10), we have a Minkowskian metric, and the length of this polygon will therefore equal the length of any polygon connecting the origin $x_{k}$ to the point $\Delta x_{k}$ in $\xi$-space, whose oriented sides are parallel to the $\xi_{\alpha}$. In particular, if the length of $e_{P_{k} P_{k+1}}$ is $\leqq \delta$ we can choose such a polygon $\lambda_{k}$ in the sphere of radius $\delta$ around $P_{k}$. If the subdivision $\pi_{m}$ was sufficiently fine we conclude from (3.7) that

$$
\left|I\left(\lambda_{k}\right)-\bar{F}\left(x_{k}, \Delta x_{k}\right)\right|<\epsilon E\left(\lambda_{k}\right),
$$

where $E\left(\lambda_{k}\right)$ is the Euclidean length of $\lambda_{k}$. Putting

$$
C_{P Q}^{*}=\sum_{k=0}^{m=1} \lambda_{k}
$$

we shall have

$$
\left|I\left(C_{P Q}^{*}\right)-\bar{P}_{m}\right|<\epsilon E\left(C_{P Q}^{*}\right) .
$$

For the sake of simple notation we shall put

$$
E(P, Q)=E\left(e_{P Q}\right), \quad I(P, Q)=I\left(e_{P Q}\right), \quad \bar{I}(P, Q)=\bar{I}\left(e_{P Q}\right) .
$$

We then have 


$$
\frac{E\left(\lambda_{k}\right)}{E\left(P_{k}, P_{k+1}\right)}=\frac{\sum p_{\alpha}\left|\xi_{\alpha}\right|}{\left|\Delta x_{k}\right|}=\frac{\sum p_{\alpha}\left|\xi_{\alpha}\right|}{\alpha\left(x_{k}, \eta\right)|\eta|} .
$$

On account of (9) we may assume that all the vectors $\xi_{1}, \cdots, \xi_{n}$ and $\eta$ are on $F\left(x_{k}, \xi\right)=1$. It follows from $\left(2.2^{\prime}\right)$ that

$$
E\left(\lambda_{k}\right) \leqq \sum p_{\alpha} \frac{1 / B}{1 / A} E\left(P_{k}, P_{k+1}\right)=\frac{A}{B} E\left(P_{k}, P_{k+1}\right) .
$$

Hence

$$
E\left(C_{P Q}^{*}\right) \leqq \frac{A}{B} E\left(C_{P Q}\right) .
$$

This, together with $\left(6^{\prime}\right)$ and (11), proves (4) and

Theorem 1. For any two points $P$ and $Q$ on $\mathfrak{M}$ one has

$$
h(P, Q)=d(P, Q) .
$$

Remark. The sums (5) can be formed for any continuous curve $C_{P Q}$. The limit of $P_{m}$ and $P_{m}^{\prime}$ ( $\infty$ admitted) will then exist and be the same for all sequences of subdivisions $\pi_{m}$ for which $\max _{k}\left(t_{k+1}-t_{k}\right) \rightarrow 0$. (6) shows that for curves $C_{P Q}$ of class $D^{\prime}$ this limit equals $I\left(C_{P Q}\right)$. Hence (5) may be used as a (generalized) definition of the F-length for continuous curves. This is done in Menger's investigations.

From these considerations we derive

THEOREM 2. On a minimizing curve $M_{P Q}$ all directions are quasi-regular.

Proof. Let $P(t), a \leqq t \leqq b$, be a representation of $M_{P Q}$ and assume there is a value $t$ for which $\alpha(x(t), \dot{x}(t))>1$. If $t$ is a point of discontinuity for $\dot{x}(t)$ then $\dot{x}_{i}(t)$ may, for instance, be the right-hand derivatives. Since $\alpha(x, \xi)$ is continuous (Theorem 2.3) we can find an interval $(t, t+\epsilon)$ such that $\alpha(x, \dot{x})>1$ in this interval. Then

$$
\int_{t}^{t+\epsilon} \bar{F}(x, \dot{x}) d t<\int_{t}^{t+\epsilon} F(x, \dot{x}) d t,
$$

and Theorem 3.1 shows that $M_{P Q}$ cannot be minimizing. Theorem 2 can be generalized to relative minimizing curves.

4. The relation between the $F$-length and the generalized length. The following consideration will be local. We may therefore restrict ourselves to a definite $K$-neighborhood $U\left(P_{0}\right)$ of a given point $P_{0}=x_{0}$. Besides 3.12 we use the notation

$$
M(C)=\int_{C} F\left(x_{0}, x^{\prime}\right) d t, \quad M(P, Q)=M\left(e_{P Q}\right) .
$$


If the indicatrix of $F(x, \xi)$ is convex at $x_{0}$ we have for any curve of class $D^{\prime}$ from $P$ to $Q$

$$
M\left(C_{P Q}\right) \geqq M(P, Q),
$$

since $M$ then is simply a Minkowskian metric for which the straight lines are minimizing. If $C_{\nu}, \nu=1,2, \cdots$, is a sequence of curves of class $D^{\prime}$ converging to $P_{0}$ we have

$$
\lim \frac{M\left(C_{v}\right)}{I\left(C_{v}\right)}=1
$$

for, on account of the continuity of $F(x, \xi)$, we have for $|\dot{x}|=1$

$$
u_{\nu}=\max _{x<C_{\nu}}\left|F\left(x_{0}, \dot{x}\right)-F(x, \dot{x})\right| \rightarrow 0
$$

and

$$
\left|\frac{M\left(C_{v}\right)-I\left(C_{v}\right)}{I\left(C_{v}\right)}\right| \leqq \frac{\int_{C_{\nu}}\left|F\left(x_{0}, x^{\prime}\right)-F(x, \dot{x})\right| d t}{I\left(C_{v}\right)} \leqq u_{v} \frac{E\left(C_{v}\right)}{B \cdot E\left(C_{v}\right)} .
$$

Let now $P_{\nu} \neq Q_{\nu}, P_{\nu} \rightarrow P_{0}, Q_{\nu} \rightarrow P_{0}$. Then we can find a curve $C_{\nu}$ of $D^{\prime}$ class from $P_{\nu}$ to $Q_{\nu}$ such that $C_{\nu} \rightarrow P_{0}$ and

$$
0 \leqq I\left(C_{\nu}\right)-d\left(P_{\nu}, Q_{\nu}\right)<\frac{E\left(P_{\nu}, Q_{\nu}\right)}{\nu} .
$$

Designating by $\xi_{\nu}$ the unit vector in the direction of $e_{P_{\nu} Q_{\nu}}$ we get on account of (1)

$$
0 \leqq \frac{I\left(C_{\nu}\right)}{M\left(C_{\nu}\right)}-\frac{d\left(P_{\nu}, Q_{\nu}\right)}{M\left(C_{\nu}\right)}<\frac{E\left(P_{\nu}, Q_{\nu}\right)}{\nu \cdot M\left(P_{\nu}, Q_{\nu}\right)}=\frac{1}{\nu \cdot F\left(x_{0}, \xi_{\nu}\right)},
$$

and therefore with the help of (2)

$$
\frac{d\left(P_{\nu}, Q_{\nu}\right)}{M\left(C_{\nu}\right)} \rightarrow 1
$$

We have, furthermore, from (1)

$$
1 \geqq \frac{d\left(P_{\nu}, Q_{\nu}\right)}{I\left(P_{\nu}, Q_{v}\right)}=\frac{d\left(P_{\nu}, Q_{\nu}\right)}{M\left(C_{\nu}\right)} \cdot \frac{M\left(C_{\nu}\right)}{M\left(P_{\nu}, Q_{\nu}\right)} \cdot \frac{M\left(P_{\nu}, Q_{\nu}\right)}{I\left(P_{\nu}, Q_{v}\right)} \geqq \frac{d\left(P_{\nu}, Q_{v}\right)}{M\left(C_{v}\right)} \cdot \frac{M\left(P_{\nu}, Q_{\nu}\right)}{I\left(P_{\nu}, Q_{v}\right)} .
$$

We see from (2), (4) that the limit of the right side is 1 ; hence $d\left(P_{\nu}, Q_{\nu}\right) / I\left(P_{\nu}, Q_{\nu}\right)$ $\rightarrow 1$. Altogether we have the

Theorem 1. If the indicatrix of $F(x, \xi)$ at $x_{0}=P_{0}$ is convex (i.e., if $F$ is quasi-regular at $\left.x_{0}\right)$, and if $P_{\nu} \rightarrow P_{0}, Q_{\nu} \rightarrow P_{0}, Q_{\nu} \neq P_{\nu}$, then 


$$
\lim \frac{d\left(P_{\nu}, Q_{\nu}\right)}{I\left(P_{\nu}, Q_{\nu}\right)}=\lim \frac{d\left(P_{\nu}, Q_{\nu}\right)}{M\left(P_{\nu}, Q_{\nu}\right)}=1 .
$$

COROLlaRy. Let $F(x, \xi)$ be quasi-regular at every point of a compact subset $\sigma$ of $a C . N$. Then for a given $\epsilon>0$ a $\delta>0$ can be found such that

$$
0 \leqq \frac{I(P, Q)}{d(P, Q)}-1<\epsilon
$$

for every pair $P, Q$ in $\sigma$ with $E(P, Q)<\delta$.

We deduce therefrom

THEOREM 2. If $F(x, \xi)$ is quasi-regular everywhere on $\mathfrak{M}$, then the F-length coincides with the generalized length for any curve $C$ of class $D^{\prime}$.

$$
I(C)=L(C) \text {. }
$$

Therefore a Hilbert arc of class $D^{\prime}$ will be minimizing.

Proof. Let $P(t), a \leqq t \leqq b$, be a representation of $C$ and let the subdivision $t_{0}=a<t_{1}<\cdots<t_{n}=b$ be chosen in such a way that the arc $C_{i}$ corresponding to the interval $t_{i} \leqq t \leqq t_{i+1}, i=0, \cdots, m-1$, is covered by one coordinate system. We have

$$
I(C)=\sum I\left(C_{i}\right), \quad L(C)=\sum L\left(C_{i}\right) .
$$

Therefore it is sufficient to prove (7) for every $i$. The set $C_{i}$ is compact. On account of the corollary we can therefore find a $\delta>0$ such that for $t_{i} \leqq \tau^{\prime}$ $<\tau^{\prime \prime} \leqq t_{i+1}$ and $\tau^{\prime \prime}-\tau^{\prime}<\delta$ one has

$$
0 \leqq I\left(P\left(\tau^{\prime}\right), P\left(\tau^{\prime \prime}\right)\right)-d\left(P\left(\tau^{\prime}\right), P\left(\tau^{\prime \prime}\right)\right)<\epsilon d\left(P\left(\tau^{\prime}\right), P\left(\tau^{\prime \prime}\right)\right) .
$$

Let now $t_{i}=\tau_{0}<\tau_{1}<\cdots<\tau_{\rho}=t_{i+1}$ be a subdivision of $\left(t_{i}, t_{i+1}\right)$ for which $\max _{i}\left(\tau_{i+1}-\tau_{i}\right)<\delta$. Then

(8) $\sum_{i=0}^{\rho-1} I\left(P\left(\tau_{i}\right), P\left(\tau_{i+1}\right)\right)-\sum_{i=0}^{\rho-1} d\left(P\left(\tau_{i}\right), P\left(\tau_{i+1}\right)\right)<\epsilon \sum_{i=0}^{\rho-1} d\left(P\left(\tau_{i}\right), P\left(\tau_{i+1}\right)\right)<\epsilon L(C)$,

and the theorem follows from (3.6) and the definition of generalized length.

If we define $F$-length and generalized length according to the Remark on page 184, formula (8) shows that (7) holds for every continuous curve $C$.

Another application of Theorem 1 is

THEOREM 3. If $x_{\nu}=P_{\nu} \rightarrow P_{0}=x_{0}$ and $\bar{x}_{\nu}=Q_{\nu} \rightarrow P_{0}$ in such a way that the univector $\xi_{\nu}=\left(\bar{x}_{\nu}-x_{\nu}\right) /\left|\bar{x}_{\nu}-x_{\nu}\right|$ converges to a vector $\xi$ (i.e., if the oriented straight line $P_{\nu} Q_{\nu}$ converges), then

$$
d\left(P_{\nu}, Q_{\nu}\right) / E\left(P_{\nu}, Q_{\nu}\right) \rightarrow \bar{F}\left(x_{0}, \xi\right) .
$$

Therefore $\overline{P Q}$ and $\bar{F}(x, \xi)$ determine each other uniquely. 
Proof $\left({ }^{3}\right) . \bar{F}\left(x_{0}, \xi\right)=1$ is convex. We know from (3.13) that $h\left(P_{\nu}, Q_{v}\right)$ $=d\left(P_{\nu}, Q_{\nu}\right)$; hence we get from (5)

$$
\frac{d\left(P_{\nu}, Q_{\nu}\right)}{\int_{e_{P \nu} Q_{\nu}} \bar{F}\left(x_{0}, x^{\prime}\right) d t}=\frac{d\left(P_{\nu}, Q_{\nu}\right)}{E\left(P_{\nu}, Q_{\nu}\right) \bar{F}\left(x_{0}, \xi_{\nu}\right)} \rightarrow 1,
$$

which proves (9).

As a last application of Theorem 1 we discuss the few differentiability properties of Hilbert arcs which hold under these general conditions.

LeMмa 1. If $F(x, \xi)$ is quasi-regular at $x_{0}=P_{0}$ and if $P_{m} \rightarrow P_{0}, Q_{m} \rightarrow P_{0}$, $R_{m} \rightarrow P_{0}$, and

$$
I_{m}=\frac{d\left(P_{m}, Q_{m}\right)+d\left(Q_{m}, R_{m}\right)}{d\left(P_{m}, R_{m}\right)} \rightarrow 1,
$$

then

$$
M_{m}=\frac{M\left(P_{m}, Q_{m}\right)+M\left(Q_{m}, R_{m}\right)}{M\left(P_{m}, R_{m}\right)} \rightarrow 1 .
$$

Proof. $d\left(Q_{m}, R_{m}\right) / d\left(P_{m}, R_{m}\right)$ is bounded, since $I_{m}$ is, and we see from Theorem 1 that

$$
\frac{d\left(Q_{m}, R_{m}\right)}{d\left(P_{m}, R_{m}\right)}\left[\frac{M\left(P_{m}, R_{m}\right)}{d\left(P_{m}, R_{m}\right)}-\frac{\dot{M}\left(Q_{m}, R_{m}\right)}{d\left(Q_{m}, R_{m}\right)}\right] \rightarrow 0 .
$$

Hence we have

$$
\begin{aligned}
\lim M_{m} & =\lim \frac{d\left(P_{m}, Q_{m}\right) M\left(P_{m}, Q_{m}\right) / d\left(P_{m}, Q_{m}\right)+d\left(Q_{m}, R_{m}\right) M\left(Q_{m}, R_{m}\right) / d\left(Q_{m}, R_{m}\right)}{d\left(P_{m}, R_{m}\right) M\left(P_{m}, R_{m}\right) / d\left(P_{m}, R_{m}\right)} \\
& =\lim I_{m}=1,
\end{aligned}
$$

We shall prove now

LEMMA 2. If under the same conditions $F(x, \xi)$ is regular at $x_{0}$ we also have

$$
E_{m}=\frac{E\left(P_{m}, Q_{m}\right)+E\left(Q_{m}, R_{m}\right)}{E\left(P_{m}, R_{m}\right)} \rightarrow 1 .
$$

Proof. By homothetic transformations for each $m$ which may carry $x$ into $\bar{x}$ we can reach

$$
\bar{P}_{m}=P_{0}, \quad E\left(\bar{P}_{m}, \bar{R}_{m}\right)=E\left(P_{0}, \bar{R}_{m}\right)=1 .
$$

We have

(3) This and the following proof follow closely the paper [3]. 


$$
\bar{I}_{m}=\frac{M\left(P_{0}, \bar{Q}_{m}\right)+M\left(\bar{Q}_{m}, \bar{R}_{m}\right)}{M\left(P_{0}, \bar{R}_{m}\right)}=I_{m}
$$

and similarly $\bar{E}_{m}=E_{m}$. If (10) did not hold we could find a sequence of subscripts $n_{1}, n_{2}, \cdots$ such that the points $\bar{Q}_{n_{i}}$ and $\bar{R}_{n_{i}}$ converge, to $\bar{Q}$ and $\bar{R}$ say, and such that $\bar{E}_{n_{i}} \rightarrow 1+\eta, \eta>0$. Then $\bar{Q}$ cannot lie on the segment $e_{P_{0} \bar{R}}$. On the other hand we should have

$$
\frac{M\left(P_{0}, \bar{Q}\right)+M(\bar{Q}, \bar{R})}{M\left(P_{0}, \bar{R}\right)}=1
$$

(on account of Lemma 1). But $F\left(x_{0}, \xi\right)$ being strictly convex this is impossible, because the Euclidean segments are the only minimizing arcs for the $M$ metric.

We can prove now

Theorem 4. Let $C_{A B}$ be a Hilbert arc from $A$ to $B$ and $P_{0}=x_{0}$ an interior point of $C_{A B}$ at which $F(x, \xi)$ is regular. If $C_{A B}$ has at $P_{0}$ a left- (right-) hand tangent, it will also have a right- (left-) hand tangent and the two tangents coincide. Hence, if $C_{A B}$ is of class $D^{\prime}$, it is of class $C^{\prime}$.

Proof. Since $C_{A B}$ is a Hilbert arc it can be mapped topologically onto an interval $a \leqq t \leqq b$. Let $P(t)$ be such a representation, and assume that $P_{0}$ corresponds to $t_{0}$ and that a left-hand tangent exists. Let $t_{v} \rightarrow t_{0}+0$. For every $t_{v}$ which is sufficiently close to $t_{0}$ we can find a value $\tau_{\nu}<t_{0}$ such that $E\left(P\left(\tau_{\nu}\right), P_{0}\right)$ $=E\left(P_{0}, P\left(t_{v}\right)\right)$. Since $C_{A B}$ is a Hilbert arc, we have

$$
\frac{d\left(P\left(\tau_{\nu}\right), P_{0}\right)+d\left(P_{0}, P\left(t_{v}\right)\right)}{d\left(P\left(\tau_{\nu}\right), P\left(t_{v}\right)\right)}=1
$$

and Lemma 2 tells us that

$$
\frac{E\left(P\left(\tau_{\nu}\right), P_{0}\right)+E\left(P_{0}, P\left(t_{v}\right)\right)}{E\left(P\left(\tau_{v}\right), P\left(t_{v}\right)\right)} \rightarrow 1 .
$$

The straight line $P\left(\tau_{\nu}\right) P_{0}$ converges to the left-hand tangent of $C_{A B}$ at $P_{0}$. Since the triangle $P\left(\tau_{\nu}\right) P_{0} P\left(\tau_{\nu}\right)$ is isosceles it follows from (11) that $\Varangle P\left(\tau_{v}\right) P_{0} P\left(t_{v}\right)$ tends to $\pi$, which proves the theorem.

In spite of this theorem, examples have been given which show that even if $F(x, \xi)$ is regular everywhere, and if all partial derivatives of $F$ with respect to the $\xi^{i}$ exist, points $A, B$ may occur for which no Hilbert arc from $A$ to $B$ of class $D^{\prime}$ exists. (See [3], pp. 281-285.)

5. The Lipschitz condition; pseudo-circles. Consider the last statement together with the example of the Minkowskian geometry, where $F$ does not depend on $x$. In the latter case no conditions regarding the dependence of $F$ on the $\xi^{i}$ are put besides the regularity, nevertheless the minimizing arcs are 
analytic curves, namely straight lines. One is led to impose on $F$ conditions regarding its variation with respect to the $x$. The weakest condition which suggests itself is the following Lipschitz condition:

To a given point $\bar{x}_{0}=\bar{P}_{0}$ there exists a C.N. $U\left(\bar{P}_{0}\right)$ such that for any two points $x$ and $\bar{x}$ in $U\left(\bar{P}_{0}\right)$

(1e) $|F(\bar{x}, \xi)-F(x, \xi)| \leqq C|\bar{x}-x| \cdot|\xi|$,

where $C$ depends on $U\left(\bar{P}_{0}\right)$ but not on $x, \bar{x}$ or $\xi$. Furthermore, we assume

(1f) The function $F(x, \xi)$ is regular everywhere.

From (1e) we derive a simple inequality which is fundamental for the following considerations:

Lemma 1. Let $e_{x_{0} x_{1}}$ be in $\bar{U}\left(\bar{P}_{0}\right)$ where $\bar{U}\left(\bar{P}_{0}\right)$ is a K.N. contained in the $U\left(\bar{P}_{0}\right)$ of the preceding definition, and let $x$ be an interior point of $e_{x_{0} x_{1}}$. Let $(x, \xi)$ and $\left(x_{1}, \xi_{1}\right)$ be two parallel vectors with

$$
\xi^{i}\left|x_{0}-x_{1}\right|=\xi_{1}^{i}\left|x_{0}-x\right|
$$

Then

$$
F\left(x_{1}, \xi_{1}\right)-F(x, \xi) \geqq \frac{|\xi|\left|x-x_{1}\right|}{\left|x-x_{0}\right|}\left\{B-C\left|x-x_{0}\right|\right\},
$$

where $B$ has the same signification as in Lemma 1.1 .

Proof. We have

$$
F\left(x_{1}, \xi_{1}\right)-F(x, \xi)=\left\{F\left(x_{1}, \xi_{1}\right)-F\left(x_{1}, \xi\right)\right\}+\left\{F\left(x_{1}, \xi\right)-F(x, \xi)\right\} .
$$

We see from (2) that

We draw from (1e) that

$$
\begin{aligned}
F\left(x_{1}, \xi_{1}\right)-F\left(x_{1}, \xi\right) & =F\left(x_{1}, \xi\right)\left[\frac{\left|x_{0}-x_{1}\right|}{\left|x_{0}-x\right|}-1\right] \\
& =F\left(x_{1}, \xi\right) \frac{\left|x-x_{1}\right|}{\left|x-x_{0}\right|} \geqq \frac{B|\xi|\left|x-x_{1}\right|}{\left|x-x_{0}\right|} .
\end{aligned}
$$

$$
\left|F\left(x_{1}, \xi\right)-F(x, \xi)\right|<C\left|x-x_{1}\right| \cdot|\xi| \text {. }
$$

These two equalities prove (3).

From now on we assume that $\mathfrak{M}$ is two-dimensional, $n=2$.

All our considerations will be restricted to a fixed convex K.N. $\bar{U}\left(\bar{P}_{0}\right)$. Then $A, B, C$ may be chosen once for all as positive constants. $k_{\rho}(P)$ may designate the circle of radius $\rho$ around $P$ and $K_{\rho}(P)$ the closed circular disk bounded by $k_{\rho}(P)$.

Let now a point $P$ and two constants $\rho$ and $P$ be chosen in such a way that 


$$
\rho<\mathrm{P}, \quad K_{\mathrm{P}}(P) \subset \bar{U}\left(\bar{P}_{0}\right) .
$$

Let $O=x_{0}$ be on $k_{\mathrm{P}}(P)$. Then $K_{\rho}(P)$ does not contain $O$. There are two continuous vector fields $(x, a)=(x, a ; O)$ in $K_{\rho}(P)$ such that the unit vector $a_{i}$ is normal to $x-x_{0}$ at $x$. Since $F(x, \xi)$ is regular everywhere, we conclude from Theorem 2.4: To $(x, a ; O)$ as covariant vector there belongs exactly one vector $(x, \xi)=(x, \xi ; O)$ with $F(x, \xi)=1$ and such that $\xi$ is supporting vector of $\gamma(x)$ to $(x, a ; O)$ as normal vector, and $(x, \xi ; O)$ will form a continuous vector field in $K_{\rho}(P)$. As $O$ traverses $k_{\mathrm{P}}(P)$ the vector field $(x, a ; O)$ will vary continuously; therefore $(x, \xi ; O)$ will depend continuously on $x$ and $O$. Using the uniform continuity we may say

LemMa 2. Given $*_{\epsilon}>0$ we can find $\rho>0$ such that

$$
\left|(x, \xi ; O)-\left(\bar{x},{ }^{*} \xi ; O\right)\right|<*^{*}
$$

for any two points $x, \bar{x}$ in $K_{\rho}(P)$ and for every $O$ on $k_{\mathrm{P}}(P)$.

Since $1 / B>|\xi|>1 / A$ the inequality (5) implies for the angle $\omega$ between $\xi$ and $* \xi$ that

$$
\omega<\arcsin \left({ }^{*} \epsilon A\right) .
$$

We designate $K_{\rho}(P)$ by $K_{\rho}^{\epsilon}(P)$ if arc $\sin \left({ }^{*} \epsilon A\right)<\frac{1}{2} \epsilon$.

We are now going to define the pseudo-circles. The basic idea of this whole last part will be that these curves have the convexity property which is expressed in Theorem 1 of this section. We keep our previous notations and fix a point $O$. Consider the differential equations

$$
\frac{d x_{i}}{d t}=\xi^{i}(x)=(x, \xi ; O), \quad i=1,2 .
$$

The solutions of (6) are called pseudo-circles with center $O$. On account of $F(x, \xi)=1$ the parameter $t$ is the $F$-length. There passes a solution of (6) through every interior point $R$ of $K_{\rho}(P)$ which can be continued to either side until it hits $k_{\rho}(P)$ the first time, at $A$ and $B$ respectively. We see

LemMA 3. Every interior point $R$ of $K_{\rho}(P)$ is contained in at least one pseudocircular arc $\sigma_{A B}$ from $A$ to $B$ of class $C^{\prime}$ and such that $A$ and $B$ are on $k_{\rho}(P)$ and all other points of $\sigma_{A B}$ are in the interior of $K_{\rho}(P)$.

If $K_{\rho}(P)$ is a $K_{\rho}^{\epsilon}(P)$ in the sense defined above, the directions of the tangents of any two pseudo-circles with the same center $O$ (at arbitrary points of the pseudo-circles) differ by less than $\frac{1}{2} \epsilon$.

Lemma 4. A Euclidean ray $r$ issuing from $O$ intersects an arc $\sigma_{A B}$ in at most one point. $\sigma_{A B}$ is therefore a simple arc.

For $r$ is never tangential to $\sigma_{A B}$ as we see from the definition and from 
$\left(2.3^{\prime}\right)$. If $r$ had more than one common point with $\sigma_{A B}$ there would be a subarc $\sigma_{C D}$ of $\sigma_{A B}$ whose endpoints are on $r$ and which has no other common points with $r$. As $x$ traverses $\sigma_{C D}$ the angle $\Varangle C O x$ must reach a maximum, for $x=x^{\prime}$ say, and the ray from $O$ through $x^{\prime}$ would be tangential to $\sigma_{A B}$ at $x^{\prime}$. It follows that $\sigma_{A B}$ has a single-valued representation $r=f(\phi)$ in polar coordinates with center $O$ which, as is shown later, is of class $C^{\prime}$. Hence the arc $\sigma_{A B}$ is simple and decomposes $K_{\rho}(P)$ into exactly two domains $\sigma_{A B}^{\prime}$ and $\sigma_{A B}^{\prime \prime}$ where $\sigma_{A B}^{\prime \prime}$ contains the points $(r, \phi)$ of $K_{\rho}(P)$ with $r<f(\phi)$ and is called the convex side of $\sigma_{A B}$ and $\sigma_{A B}^{\prime}$ contains the points $(r, \phi)$ with $r>f(\phi)$ and is called the concave side of $\sigma_{A B}$.

LeMma 5. Let $R$ be a given interior point of $K_{\rho}(P)$ and $\xi$ a given direction through $R$. There is always a pseudo-circular arc $\sigma_{A B}$ through $R$ with tangent $\xi$ at $R=x_{r}$ and such that $\sigma_{A B}^{\prime}$ is on the left- (right-) side of $\sigma_{A B}$ with respect to the orientation of $\sigma_{A B}$.

Proof. Let $F\left(x_{r}, \xi\right)=1$. Let $t$ be a supporting line of $F\left(x_{r}, \xi\right)=1$ at $\xi$ and $a_{i}$ the unit vector normal to $t$. Then $\left(x_{r}, a\right)$ will be a supporting normal to $\xi_{i}$ as supporting vector. Take the straight line perpendicular to $a_{i}$ through $R$ and let $O, O^{\prime}$ be its intersections with $k_{\mathrm{P}}(P)$. The pseudo-circles through $R$ with centers $O$ and $O^{\prime}$ have a tangent with direction $\xi$ at $R$ and the corresponding concave domain is to the right or left of the pseudo-circle according to whether $O$ or $O^{\prime}$ was taken.

We now come to the main property of the pseudo-circles.

Theorem 1. Let $E(O, P)=\mathrm{P}, \rho<\mathrm{P}, K_{\mathrm{P}}(P) \subset \bar{U}\left(P_{0}\right)$ and $\rho+\mathrm{P}<B / 2 C$. Let $\sigma_{A B}$ be a pseudo-circular arc decomposing $K_{\rho}(P)$ into the concave side $\sigma_{A B}^{\prime}$ and the convex side $\sigma_{A B}^{\prime \prime}$. Let $R$ and $S$ be interior points of $\sigma_{A B}$ and such that $R$ precedes $S$ on $\sigma_{A B}$. If $\sigma_{R S}$ designates the subarc from $R$ to $S$ of $\sigma_{A B}$ and $C_{R S}$ is any continuous curve from $R$ to $S$ which, except for $R$ and $S$ is completely in the interior of $\sigma_{A B}^{\prime}$ then

$$
L\left(C_{R S}\right)>L\left(\sigma_{R S}\right)=I\left(\sigma_{R S}\right) .
$$

Proof. Since $\sigma_{R S}$ is rectifiable, we may assume that $C_{R S}$ is rectifiable and also that it is a simple arc. Let $P(t), a \leqq t \leqq b$, be a representation of $C_{R S}$. Introduce again polar coordinates $(r, \phi)$ with center $O=x_{o}$, let $P(x)=(r(t), \omega(t))$, and let $\sigma_{A B}$ again have the representation $r=f(\phi)$, in particular $\left(f\left(\phi_{1}\right), \phi_{1}\right)=R$, $\left(f\left(\phi_{2}\right), \phi_{2}\right)=S$. We may assume that $\phi_{1}<\phi_{2}$. The function $\omega(t)$ is continuous; therefore there is a last value $t_{1}$ in $(a, b)$ for which $\omega\left(t_{1}\right)=\phi_{1}$ and a first value $t_{2}$ in $\left(t_{1}, b\right)$ such that $\omega\left(t_{2}\right)=\phi_{2}\left(t_{1}=a\right.$ or $t_{2}=b$ admitted $)$.

We then have

$$
\phi_{1}<\omega(t)<\phi_{2} \quad \text { for } t_{1}<t<t_{2} .
$$

Put $P\left(t_{1}\right)=R_{1}, P\left(t_{2}\right)=S_{1}$ and let $C_{R_{1} S_{1}}$ be the subarc $t_{1} \leqq t \leqq t_{2}$ of $C_{R S}$. It is 
sufficient to prove that

$$
I\left(C_{R_{1} S_{1}}\right)>L\left(\sigma_{R S}\right) .
$$

It follows from (8) that for $t_{1} \leqq t \leqq t_{2}$ the segment $e_{O P(t)}$ will intersect $\sigma_{A B}$ in exactly one point, namely ${ }_{*} P(t)=(f(\omega(t)), \omega(t))$ which we call the projection of $P(t)$ on to $\sigma_{A B}$. We have ${ }_{*} P\left(t_{1}\right)=R,{ }_{*} P\left(t_{2}\right)=S$. Let $t^{\prime}-t_{1}>0$ and $t_{2}-t^{\prime \prime}>0$ be so small that ${ }_{*} P\left(t^{\prime}\right)$ precedes ${ }_{*} P\left(t^{\prime \prime}\right)$ on $\sigma_{A B}$ and put $P\left(t^{\prime}\right)=R^{\prime}, P\left(t^{\prime \prime}\right)=S^{\prime}$. For $t^{\prime} \leqq t \leqq t^{\prime \prime}$ the number $E\left(P(t),{ }_{*} P(t)\right)$ is a positive and continuous function of $t$, which therefore has a positive maximum $\alpha$. Let now

$$
0<3 \epsilon<\frac{\alpha \gamma C}{2}
$$

with $\gamma=(\mathrm{P}-\rho)\left|\omega\left(t^{\prime}\right)-\omega\left(t^{\prime \prime}\right)\right|$. We choose $t_{1}<t_{*}<t^{\prime}$ and $t^{\prime \prime}<t^{*}<t_{2}$ such that with $\bar{R}=P\left(t_{*}\right), \bar{S}=P\left(t^{*}\right)$ we have

$$
L\left(C_{R_{1} S_{1}}\right)-L\left(C_{\dot{R} \bar{S}}\right)<\epsilon, \quad I\left(\sigma_{R S}\right)-I\left(\sigma_{*} \bar{R}_{*} \bar{S}\right)<\epsilon .
$$

We then choose a subdivision $\pi=\left(\tau_{0}=t_{*}<\tau_{1}<\cdots<\tau_{m}=t^{*}\right)\left(P\left(\tau_{i}\right)=P_{i}\right)$ such that $t^{\prime}$ and $t^{\prime \prime}$ occur among the $\tau_{i}$ and so fine that firstly the chords $e_{P_{i} P_{i+1}}$ are all contained in the interior of $\sigma_{A B}^{\prime}$ and secondly

$$
\left|L\left(C_{\ddot{R} \bar{S}}\right)-\sum_{\nu=0}^{m-1} I\left(P_{\nu}, P_{\nu+1}\right)\right|<\epsilon
$$

which is possible on account of the Remark on page 184 and thirdly

$$
E\left(P,{ }_{*} P\right)>\frac{1}{2} \alpha \quad \text { for } P \subset \sum e_{P_{\nu} P_{\nu+1}} \text {. }
$$

Let ${ }_{*} P_{\nu}$ be the projection of $P_{\nu}$. We call the subarc $\sigma_{*} P_{\nu *} P_{\nu+1}$ of $\sigma_{A B}$ regular if ${ }_{*} P_{\nu}$ precedes ${ }_{*} P_{\nu+1}$ on $\sigma_{A B}$. The regular arcs $\sigma_{* P_{\nu} P_{\nu+1}}$ cover $\sigma_{*} \bar{R}_{*} \bar{S}$, hence designating by $\sum$ ' the summation over the regular subarcs,

$$
\sum^{\prime} I\left(\sigma_{* P_{\nu} * P_{\nu+1}}\right) \geqq I\left(\sigma_{*} \bar{R}_{*} \bar{S}_{*}\right)
$$

Let

$$
* x_{\nu}(t), \quad t_{\nu} \leqq t \leqq t_{\nu+1},
$$

be a representation of the regular arc $\sigma_{* P_{\nu} P_{\nu+1}}$, where ${ }_{*} x_{\nu}(t)$ is of class $C^{\prime}$. To every point $* x(t)$ there belongs exactly one point $x(t)$ of $e_{P_{y} P_{\nu+1}}$ whose projection $* x(t)$ is. In this way $x(t)$ is defined as function of the same parameter $t$. $x_{i}(t)$ is also of class $C^{\prime}$. (One sees this immediately if one puts $x(t)=(r(t)$, $\phi(t))$, for then

$$
\phi(t)=\operatorname{arctg} \frac{*^{x_{2}}(t)-x_{02}}{{ }_{*} x_{1}(t)-x_{01}}
$$

where $x_{0}=0$.) 
We form

$$
\begin{aligned}
I\left(P_{\nu}, P_{\nu+1}\right)-I\left(\sigma_{* P_{\nu} * P_{\nu+1}}\right)= & \int_{t_{\nu}}^{t_{\nu+1}}\left\{F(x(t), \dot{x}(t))-F\left({ }_{*} x(t),{ }_{*} \dot{x}(t)\right)\right\} d t \\
= & \int_{t_{\nu}}^{t_{\nu+1}}\left\{F(x, \dot{x})-F\left({ }_{*} x, \kappa \dot{x}\right)\right\} d t \\
& +\int_{t_{\nu}}^{t_{\nu+1}}\left\{F\left({ }_{*} x, \kappa \dot{x}\right)-F\left({ }_{*} x,{ }_{*} x^{\prime}\right)\right\} d t,
\end{aligned}
$$

where $\kappa=\left|* x-x_{0}\right| /\left|x-x_{0}\right|$. By construction the vector $\pm\left\{\kappa\left[x_{i}(t+\Delta)-x_{i}(t)\right]\right.$ $\left.-\left[x_{i}(t+\Delta)-_{*} x_{i}(t)\right]\right\}$ is either a zero vector or has the direction of the segment $e_{x_{0 *} x}(t+\Delta)$. Letting $\Delta \rightarrow 0$ we see that $\pm\left(\kappa \dot{x}_{i}(t)-{ }_{*} \dot{x}_{i}(t)\right)$ has the direction of $e_{x_{0}, x(t)}$. Furthermore, it follows from the definition of pseudo-circles that $* \dot{x}(t)$ is a supporting vector of $F\left({ }_{*} x, \xi\right)=1$ belonging to a covariant vector $a_{i}$ perpendicular to the ray $\overrightarrow{O_{*}} \vec{x}$. Let $A_{i}$ be the normalized normal in the direction $a_{i}$. Applying (2.6) we see that

$$
F\left({ }_{*} x, \kappa \dot{x}\right)-F\left({ }_{*} x,{ }_{*} \dot{x}\right) \geqq A_{i}\left(\kappa \dot{x}_{i}-{ }_{*} \dot{x}_{i}\right) .
$$

Hence the second integral on the right side of (15) is not negative. Only for this conclusion we used the defining property of pseudo-circles.

The Lipschitz condition will be used to get an estimate of the first integral. For we get from (3) that

$$
\begin{aligned}
F(x, \dot{x})-F\left({ }_{*} x, \kappa \dot{x}\right) & \geqq \frac{\kappa|\dot{x}| \cdot\left|x-{ }_{*} x\right|}{\left|{ }_{*} x-x_{0}\right|}\left\{B-C\left|{ }_{*} x-x_{0}\right|\right\} \\
& =\frac{|\dot{x}| \cdot\left|x-{ }_{*} x\right|}{\left|x-x_{0}\right|}\left\{B-C\left|{ }_{*} x-x_{0}\right|\right\} .
\end{aligned}
$$

Now we have $\left|* x-x_{0}\right|<B /(2 C),\left|x-x_{0}\right|<B /(2 C)$. Therefore

$$
I\left(P_{\nu}, P_{\nu+1}\right)-I\left(\sigma_{* P_{\nu} * P_{\nu+1}}\right) \geqq 0
$$

for the regular arcs on $C_{\bar{R} \bar{S}}$ and on account of (13) we have $|x-* x|>\frac{1}{2} \alpha$ for the regular arcs on $C_{R^{\prime} S^{\prime}}$. Hence we have for all these arcs, using the above inequalities for $\left|* x-x_{0}\right|$ and $\left|x-x_{0}\right|$,

$$
F(x, \dot{x})-F\left({ }_{*} x, \kappa \dot{x}\right) \geqq|\dot{x}| \frac{\alpha C}{2} .
$$

We then get from (15) that

$$
I\left(P_{\nu}, P_{\nu+1}\right)-I\left(\sigma_{* P_{\nu} * P_{\nu+1}}\right) \geqq \int_{t_{\nu}}^{t_{\nu+1}}|\dot{x}| \frac{\alpha C}{2} d t=\frac{\alpha C}{2} E\left(P_{\nu}, P_{\nu+1}\right),
$$

and therefrom, using (14), 


$$
\begin{aligned}
\sum I\left(P_{\nu}, P_{\nu+1}\right) & \geqq \sum^{\prime} I\left(P_{\nu}, P_{\nu+1}\right) \geqq \sum^{\prime} I\left(\sigma_{* P_{\nu} * P_{\nu+1}}\right)+\sum^{\prime \prime} \frac{\alpha C}{2} E\left(P_{\nu}, P_{\nu+1}\right) \\
& \geqq I\left(\sigma_{* \vec{R}_{*} \bar{S}}\right)+\frac{\alpha C}{2} \sum^{\prime \prime} E\left(P_{\nu}, P_{\nu+1}\right),
\end{aligned}
$$

where $\sum^{\prime \prime}$ means the sum over all regular $e_{P_{\nu} P_{\nu+1}}$ for which $P_{\nu}$ and $P_{\nu+1}$ belong to $C_{R^{\prime} S^{\prime}}$.

To get an estimate for $\sum^{\prime \prime} E\left(P_{\nu}, P_{\nu+1}\right)$ we remark that the $e_{P_{\nu} P_{\nu+1}}$ are outside of $K_{\mathrm{P}-\rho}(O)$. Therefore

$$
E\left(P_{\nu}, P_{\nu+1}\right)>\left|\omega\left(\tau_{\nu+1}\right)-\omega\left(\tau_{\nu}\right)\right|(\mathrm{P}-\rho),
$$

and since the projections of the regular segments $e_{P_{\nu} P_{\nu+1}}$ cover the arc of $K_{\mathrm{P}-\rho}(0)$ from $\omega\left(t^{\prime}\right)$ to $\omega\left(t^{\prime \prime}\right)$ we have

$$
\sum^{\prime \prime} E\left(P_{\nu}, P_{\nu+1}\right)>\left|\omega\left(t^{\prime}\right)-\omega\left(t^{\prime \prime}\right)\right| \cdot(\mathrm{P}-\rho) .
$$

Altogether we get from (10), (11), (12), (16) and (17) that

$$
L\left(C_{R_{1} S_{1}}\right)-I\left(\sigma_{R S}\right)>\frac{\alpha \gamma C}{2}-3 \epsilon>0,
$$

which proves the theorem.

Since $C_{R_{1} S_{1}}$ instead of $C_{R S}$ occurs in the last inequality, we can pronounce the following result.

TheOREM 1'. Let $\rho$ and $\mathrm{P}$ satisfy the conditions of Theorem 1. Let $\Sigma_{A B}$ be a simple oriented arc which, except for its endpoints $A, B$ is in the interior of $K_{\rho}(P)$. Let $\Sigma_{A B}$ contain a pseudo-circular subarc $\sigma_{A^{\prime} B^{\prime}}$ whose orientation coincides with that of $\Sigma_{A B}$. Designate by $\Sigma_{A B}^{\prime}, \Sigma_{A B}^{\prime \prime}$ the sets into which $\Sigma_{A B}$ decomposes $K_{\rho}(P)$, let $\Sigma_{A B}^{\prime}$ be the one whose points close to interior points of $\sigma_{A^{\prime} B^{\prime}}$ are on the concave side of $\sigma_{A^{\prime} B^{\prime}}$ and finally let $R$ and $S$ be two points of $\sigma_{A^{\prime} B^{\prime}}$ where $R$ precedes $S$. Then (7) will hold for any continuous arc $C_{R S}$ from $R$ to $S$ which, except for $R$ and $S$, is in the interior of $\Sigma_{A B}^{\prime}$.

6. Proof that every Hilbert arc is of class $C^{\prime}$. In this section we are going to prove that under the conditions (1a)-(1f) every Hilbert arc is of class $C^{\prime}$. We shall see first that a Hilbert arc from $Q$ to $P$ has a tangent at $P$; i.e., we shall prove

Theorem 1. A simple continuous arc $C_{Q P}$ from $Q$ to $P$ which has no tangent at $P$ is no Hilbert arc.

Proof. Since $C_{Q P}$ has no tangent at $P$ the rays $\overrightarrow{R P}$ must have at least two different accumulation directions $-g_{1}$ and $-g_{2}$ as $R$ tends to $P$ on $C_{Q P}$. Let $\phi$ and $\psi$ be the two angles between $+g_{1}$ and $+g_{2}$ so that $\phi+\psi=2 \pi$. By $\omega_{\eta}(h, T)$ we designate generally a half-angle with $h$ as bisector, opening $\eta$ and vertex $T$. 
Let now $+g$ and $-g$ be the bisectors of $\phi$ and $\psi$ respectively, and choose $\eta$ and $\epsilon$ such that the 4 angles

$$
\omega_{\eta}\left(+g_{1}, P\right), \omega_{\eta}\left(+g_{2}, P\right), \omega_{3 \epsilon}\left(+g_{1}, P\right), \omega_{3 \epsilon}(-g, P)
$$

are disjoint except for their common vertex $P$.

Take now a circle $K_{\rho}^{\epsilon}(P)$ (cf. p. 190, after $\left(5^{\prime}\right)$ ), and let $C_{Q P}$ be represented by $P(t), a \leqq t \leqq b$, and let $t_{\rho}$ be the greatest number for which $E(P(t), P)=\rho$. Call $C_{\rho}$ the subarc $t_{\rho} \leqq t \leqq b$ of $C_{Q P}$. There is a subarc $C_{P_{1} P_{2}}$ of $C_{\rho}$ which connects a point $P_{1}$ of $\omega_{\eta}\left(+g_{1}\right)$ to a point $P_{2}$ of $\omega_{\eta}\left(+g_{2}\right)$ and which is completely in either

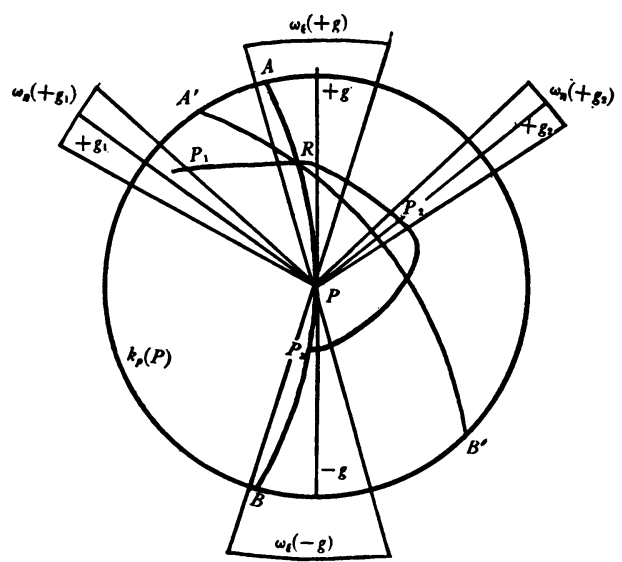

FIG. 1

$\omega_{\phi+\eta}(+g, P)$ or $\omega_{\psi+\eta}(-g, P)$. Assume, for instance, that $C_{P_{1} P_{2}} \subset \omega_{\phi+\eta}(+g)$. We then construct a pseudo-circular arc $\sigma_{A B}$ with center on $k_{\mathrm{P}}(P)$ which passes through $P$, has $-g$ as tangent at $P$ and for which $P_{1}$ is in $\sigma_{A B}^{\prime \prime}$ and $P_{2}$ in $\sigma_{A B}^{\prime}$. This is possible on account of Lemma 5.4 and because $\sigma_{A B}$ is contained in $\omega_{\epsilon}(+g, P)+\omega_{\epsilon}(-g, P)$ (the tangent of $\sigma_{A B}$ varies in $K_{\rho}(P)$ by less than $\left.\frac{1}{2} \epsilon\right)$. Therefore $C_{P_{1} P_{2}}$ intersects $\sigma_{A B}$. Among the intersections there is one, $R$ say, such that $C_{R P_{2}}$ belongs to $\sigma_{A B}^{\prime}$ except for $R$. We now draw through $R$ a pseudocircular arc $\sigma_{B^{\prime} A^{\prime}}$ with center $O^{\prime}$ on $k_{\rho}(P)$ with the following properties: The tangent of $\sigma_{B^{\prime} A}$, at $R$ forms with $+g$ the angle $\epsilon$ and such that it points into $\sigma_{A B}^{\prime \prime}$, furthermore such that if $\sigma_{A B}^{\prime}$ is on the left (right) of $\sigma_{A B}$, the set $\sigma_{B^{\prime} A^{\prime}}^{\prime}$ is on the right (left) of $\sigma_{B^{\prime} A^{\prime}}$. The subarc $\sigma_{R A^{\prime}}$ of $\sigma_{B^{\prime} A^{\prime}}$ will lie in $\sigma_{A B}^{\prime \prime}$ except for $R$. Call ${ }^{*} \sigma_{A^{\prime} R}$ the arc $\sigma_{R A^{\prime}}$ in opposite orientation. Then the arc $\Sigma_{A^{\prime} B}=*_{\sigma_{A^{\prime} R}}+\sigma_{R B}$ will be simple. $\omega_{\eta}\left(+g_{1}\right)$ and $\omega_{\eta}\left(+g_{2}\right)$ are on different sides of $\Sigma_{A^{\prime} B}$ since $\Sigma_{A^{\prime} B}$ lies in $\omega_{3 \epsilon}(+g, P)+\omega_{3 \epsilon}(-g, P)$. The arc $C_{P_{2} P}$ contains points of $\omega_{\eta}\left(+g_{1}, P\right)$; therefore traversing $C_{P_{2} P}$ we hit $\Sigma_{A^{\prime} B}$ the first time, at $P_{3}$ say. Then $C_{R P_{3}}$ does not intersect $\Sigma_{A^{\prime} B}$ except at $R$ and $P_{3}$ because ${ }^{*} \sigma_{A^{\prime} R}$ is contained in $\sigma_{A B}^{\prime \prime}$ and $C_{R P_{2}}$ in $\sigma_{A B}^{\prime}$. If $P_{3} \subset \sigma_{R A}$, we see from Theorem $5.1^{\prime}$ that 


$$
d\left(R, P_{3}\right) \leqq I\left(\sigma_{R P_{3}}\right)<L\left(C_{R P_{3}}\right),
$$

where $\sigma_{R P_{3}}$ is the subarc from $R$ to $P_{3}$ of $\sigma_{B^{\prime} A^{\prime}}$ and if $P_{3} \subset \sigma_{A B}$ we see from the same theorem that

$$
d\left(R, P_{3}\right) \leqq I\left(\sigma_{R P_{3}}\right)<L\left(C_{R P_{3}}\right),
$$

where $\sigma_{R P_{3}}$ is the subarc of $\sigma_{A B}$ from $R$ to $P_{3}$. Therefore in neither case can $C_{P Q}$ be a Hilbert arc, q.e.d.

Let now $H_{Q P}$ be a Hilbert arc from $Q$ to $P$. Then the subarc $H_{Q R}$ of $H_{Q P}$ is a Hilbert arc for every interior point $R$ of $H_{Q P}$. It therefore has a tangent at $R$. It then follows from Theorem 4.4 that $H_{Q P}$ has a tangent at $R$. For the point $Q$ we can prove the existence of a tangent as follows.

If $H_{P Q}^{*}$ is $H_{Q P}$ in opposite orientation, it is a Hilbert arc from $P$ to $Q$ with respect to $F^{*}(x, \xi)=F(x,-\xi)$. Therefore $H_{P Q}^{*}$ has a tangent at $Q$; hence $H_{Q P}$ has one too. We see

TheOREM 2. A Hilbert arc has a tangent everywhere.

We shall prove now

TheOREM 3. An arc $C_{Q P}$ from $Q$ to $P$ which has a tangent everywhere, but whose tangent is not continuous at $P$, is no Hilbert arc.

Proof. Let $\beta$ be the direction of the tangent at $P$. Then a sequence of points $P_{m} \rightarrow P$ on $C_{Q P}$ exists such that the directions $\gamma_{m}$ of the tangent of $C_{Q P}$ at $P_{m}$ converge to a line with direction $\gamma \neq \beta$. Let $\phi$ be the lesser of the two angles formed by $\beta$ and $\gamma$ at $P$ and let $\xi$ be a bisector of this angle. Consider $K_{\rho}^{\phi / 2}(P)$ (for definition see page 190). On $k_{\mathrm{P}}(P)$ we choose the point $O$ such that the vector field $(x, \xi ; O)$ has the direction $\xi$ at $P$ and such that if $\sigma_{A B}$ is a pseudocircular arc of the vector field the direction $\gamma$ points into $\sigma_{A B}^{\prime}$.

Let $\sigma_{A_{m} B_{m}}$ be a pseudo-circular arc with center $O$ through $P_{m}$. Then its direction at $P_{m}$ differs from $\xi$ by less than $\phi / 4$, and since $\xi$ bisects $\Varangle \beta \gamma$ and $\gamma_{m} \rightarrow \gamma$ and $\vec{P}_{m} \vec{P}=\beta_{m} \rightarrow \beta$ we see that for large $m$ the rays $\gamma_{m}$ and $\beta_{m}$ will not belong to the angles $\omega_{\phi / 2}\left(-{ }^{*} \xi, P_{m}\right)$ or $\omega_{\phi / 2}\left(+^{*} \xi, P_{m}\right)$, where ${ }^{*} \xi$ designates the parallel to $\xi$ through $P_{m}$. Therefore $\gamma_{m}$ will point into the concave side $\sigma_{A_{m} B_{m}}^{\prime}$ of $\sigma_{A B}$ and $\beta_{m}$ in to $\sigma_{A_{m} B_{m}}^{\prime \prime}$.

As in the preceding proof we now replace the subarc $\sigma_{A_{m} P_{m}}$ of $\sigma_{A_{m} B_{m}}$ by another pseudo-circular arc $\sigma_{P_{m} A^{\prime}}$ which is a subarc of a pseudo-circular arc $\sigma_{B^{\prime} A^{\prime}}$ through $P_{m}$ with center $O^{\prime} \subset K_{\rho}(P)$ where $O^{\prime}$ is determined as follows: The field vector of $\left(x, \xi ; O^{\prime}\right)$ at $P$ has direction $-\xi$ and $\sigma_{B^{\prime} A}^{\prime}$, is on the right (left) of $\sigma_{B^{\prime} A^{\prime}}$ if $\sigma_{A_{m} B_{m}}^{\prime}$ is on the left (right) of $\sigma_{A_{m} B_{m}}$. Then $\gamma$ will still point into the concave side of any pseudo-circular arc of $K_{\rho}^{\phi / 2}(P)$ with center $O^{\prime}$.

Call $\sigma_{A^{\prime} P_{m}}^{*}$ the subarc $\sigma_{P_{m} A^{\prime}}$ of $\sigma_{B^{\prime} A^{\prime}}$ in opposite orientation. Then

$$
\Sigma_{A^{\prime} B_{m}}={ }^{*} \sigma_{A}{ }^{\prime} P_{m}+\sigma_{P_{m} B_{m}}
$$

will be a simple arc, which is contained in the angles $\omega_{\phi / 2}\left({ }^{*} \xi, P_{m}\right)$ and 
$\omega_{\phi / 2}\left(-* \xi, P_{m}\right)$. Therefore $\gamma_{m}$ and $\beta_{m}$ point into different sides $\Sigma_{1}$ and $\Sigma_{2}$ of $\Sigma_{A^{\prime} B_{m}}$.

Let $\gamma_{m}$ point in to $\Sigma_{1}$.

Now $C_{P Q}$ has tangent $\gamma_{m}$ in $P_{m}$. Therefore the points on $C_{P Q}$ following $P_{m}$ and sufficiently close to $P_{m}$ must be in $\Sigma_{1}$. Since $\beta_{m}$ contains $P$, the point $P$ must be in $\Sigma_{2}$. Therefore, traversing $C_{Q P}$ from $P_{m}$ on we shall meet $\Sigma_{A^{\prime} B_{m}}$ a first time, at $R$ say. Then one sees, as in the preceding proof that, no matter whether $R$ belongs to $\sigma_{P_{m} A^{\prime}}$ or $\sigma_{P_{m} B}$ the arc $C_{P_{m} R}$ will be longer than the corresponding subarc of $\sigma_{P_{m} A^{\prime}}$ or $\sigma_{P_{m} B}$. We see, as in the preceding proof, that $H_{Q P}$ has a continuous tangent at $Q$ too, and hence that every Hilbert arc $H_{Q P}$ has a continuous tangent everywhere. If we then introduce the arc length $s$ on $H_{Q P}$ as parameter, $H_{Q P}$ will have a representation $x_{i}(s)$ where the $x_{i}(s)$ are of class $C^{\prime}$. Therefore $H_{Q P}$ is of class $C^{\prime}$. We have

THEOREM 4. If the space $\mathfrak{M}$ is two-dimensional and if the conditions (1a)(1f) hold, every Hilbert arc is of class $C^{\prime}$.

7. Examples. Finally we discuss the question whether under the conditions of the last theorem any of the following questions can be answered positively:

(1) Have the minimizing curves second derivatives $\left({ }^{4}\right)$ ?

(2) Is there at least one minimizing curve through a given line element?

(3) Is here at most one minimizing curve through a given line element?

The answer to all three questions is negative. In order to construct a simple example we remind the reader of the function $F(x, y ; \xi, \eta)=\left(\xi^{2}+\eta^{2}\right)^{1 / 2} / y$, which defines a hyperbolic metric in the half-plane $y>0$ of the Euclidean $(x, y)$ plane. Therefore

$$
\frac{\left(\xi^{2}+\eta^{2}\right)^{1 / 2}}{y+1}, \quad \frac{\left(\xi^{2}+\eta^{2}\right)^{1 / 2}}{1-y}
$$

will define hyperbolic metrics in the half-planes $y>-1$ and $y<1$ respectively.

The integrand

$$
F(x, y ; \xi, \eta)=\frac{\left(\xi^{2}+\eta^{2}\right)^{1 / 2}}{1+|y|}
$$

is regular everywhere and we have

$$
\begin{aligned}
|F(\bar{x}, \bar{y} ; \xi, \eta)-F(x, y ; \xi, \eta)| & =\left(\xi^{2}+\eta^{2}\right)^{1 / 2} \cdot\left|\frac{1}{1+|\bar{y}|}-\frac{1}{1+|y|}\right| \\
& \leqq(|y|-|\bar{y}|) \cdot\left(\xi^{2}+\eta^{2}\right)^{1 / 2} \\
& \leqq\left((x-\bar{x})^{2}+(y-\bar{y})^{2}\right)^{1 / 2} \cdot\left(\xi^{2}+\eta^{2}\right)^{1 / 2}
\end{aligned}
$$

(4) This question has an invariant meaning only if $\mathfrak{M}$ is of class 2 , which will be the case in the following examples. 
so that the Lipschitz condition (c) is uniformly satisfied. For $y \geqq 0(y \leqq 0)$ the minimizing arcs are the semicircles perpendicular to $y=-1(y=1)$. Therefore every point on $y=0$ can be joined to $(0,0)$ by exactly two minimizing arcs. Hence no minimizing curve with $y=0$ as tangent of $(0,0)$ exists. The same holds for every other point on the $x$-axis. This answers (2) negatively.

To get examples for the other two questions we consider

$$
F(x, y ; \xi, \eta)=\frac{\left(\xi^{2}+\eta^{2}\right)^{1 / 2}}{1-|y|} .
$$

Here the Lipschitz condition (c) holds uniformly for $|y| \leqq r<1$ therefore in a suitable neighborhood of any given point in $|y|<1$. For $y>0 \quad(y<0)$ the extremals are now the semicircles perpendicular to $y=1(y=-1)$. Since we have at every point $(a, 0)$ of the $x$-axis one circle of each family tangent to the $x$-axis at $(a, 0)$, we see that question (3) has a negative answer.

But this example also settles question (1). Obviously the segment of the $x$-axis joining $(0,0)$ to $(a, 0)$ is the only minimizing arc connecting them. Take now a point $\left(a, 1-1 / 2^{1 / 2}\right)$ with $a>1 / 2^{1 / 2}$ and let $H$ be a Hilbert arc connecting $\left(a, 1-1 / 2^{1 / 2}\right)$ to $(0,0)$. It cannot contain points $(\bar{x}, \bar{y})$ with $\bar{y}<0$ for then it would contain a point $(b, 0)$ preceding $(\bar{x}, \bar{y})$ and the segment from $(b, 0)$ to $(0,0)$ would be shorter than the corresponding arc of $H$.

We know from Theorem 6.4 that $H$ is of class $C^{\prime}$. It follows therefrom that $H$ must look as follows: Let $C$ be the semicircle perpendicular to $y=+1$ through $\left(a, 1-1 / 2^{1 / 2}\right)$ which is tangent to the $x$-axis, at $\left(a-1 / 2^{1 / 2}, 0\right)$. Then the arc of $c$ from $\left(a, 1-1 / 2^{1 / 2}\right)$ to $\left(a-1 / 2^{1 / 2}, 0\right)$ together with the segment of the $x$-axis between $\left(a-1 / 2^{1 / 2}, 0\right)$ and $(0,0)$ will constitute $H$, but $H$ has no second derivative at $\left(a-1 / 2^{1 / 2}, 0\right)$.

The family of minimizing curves issuing from $(0,0)$ with the $x$-axis as tangent does not form a field. We were not able to find a counter-example for (3) with the field property.

\section{REFERENCES}

1. O. Bolza, Vorlesungen über Variationsrechnung, Leipzig, 1909.

2. T. Bonnesen, and W. Fenchel, Theorie der konvexen Körper, Berlin, 1934.

3. H. Busemann, Lokale Eigenschaften der zu Variationsproblemen gehörigen metrischen Räume, Fundamenta Mathematicae, vol. 32 (1939), pp. 265-287.

4. H. Busemann, and Willy Feller, Krümmungseigenschaften konvexer Flächen, Acta Mathematica, vol. 66 (1935), pp. 1-47.

Illinois Institute of Technology, Chicago, Ill.

Institute for Advanced Study, Princeton, N. J. 